\title{
Gas Classification Using Combined Features Based on a Discriminant Analysis for an Electronic Nose
}

\author{
Sang-Il Choi, ${ }^{1}$ Taekyu Eom, ${ }^{1}$ and Gu-Min Jeong ${ }^{2}$ \\ ${ }^{1}$ Department of Computer Science and Engineering, Dankook University, 126 Jukjeon-dong, Suji-gu, Yongin-si, \\ Gyeonggi-do 448-701, Republic of Korea \\ ${ }^{2}$ Electrical Engineering, Kookmin University, 861-1, Jeongneung-dong, Seongbuk-gu, Seoul 136-702, Republic of Korea
}

Correspondence should be addressed to Gu-Min Jeong; gm1004@kookmin.ac.kr

Received 5 October 2015; Revised 5 February 2016; Accepted 3 March 2016

Academic Editor: Sara Casciati

Copyright (C) 2016 Sang-Il Choi et al. This is an open access article distributed under the Creative Commons Attribution License, which permits unrestricted use, distribution, and reproduction in any medium, provided the original work is properly cited.

\begin{abstract}
This paper proposes a gas classification method for an electronic nose (e-nose) system, for which combined features that have been configured through discriminant analysis are used. First, each global feature is extracted from the entire measurement section of the data samples, while the same process is applied to the local features of the section that corresponds to the stabilization, exposure, and purge stages. The discriminative information amounts in the individual features are then measured based on the discriminant analysis, and the combined features are subsequently composed by selecting the features that have a large amount of discriminative information. Regarding a variety of volatile organic compound data, the results of the experiment show that, in a noisy environment, the proposed method exhibits classification performance that is relatively excellent compared to the other feature types.
\end{abstract}

\section{Introduction}

An electronic nose (e-nose) is an electronic system that uses a sensor to classify the types of specified gas [1-4]. As it becomes easily tired, the human olfactory function does not recognize different smells continuously, and it is therefore limited in the types of smells that it can recognize. In contrast, an e-nose can monitor smells constantly and this capability is especially advantageous to humans in terms of the detection of harmful gases $[5,6]$. For early e-nose systems, a calorimetric sensor was used to express the measured gas values in the form of a color array [7]; additionally, this system requires the completion of a number of complex analyses, as well as precision equipment such as a gas-chromatography system for gas classification or a mass spectrometer that combines complex machine intelligences, thereby limiting the service environment $[8,9]$. In terms of its real-world application, the calorimetric-sensor-based system, it is therefore limited to the specific applications; however, with the development of electrochemical sensors and IT (both hardware and software), electronic systems became more intelligent and portable $[10,11]$. Due to these technological developments, an increasingly diverse array of e-nose applications continues to gradually emerge and includes medical check-ups [12], pollution and gas leak searches [13], environmental protection [14], and cosmetics industry $[4,15,16]$.

The e-nose system is composed of sensor arrays that consist of multiple channels and computing systems [2, 17]. Since each channel of the sensor array has a different characteristic, it responds differently depending on the type (class) of gas, and an electronic interface converts the sensor array response into vector-type numerical data. In the computing system, the features that are useful for the gas are extracted from the converted data, and a classifier is used to discern the gas classes. Sensor array sensitivity, feature extraction, and classifier performance are all important factors in a performance assessment of an e-nose system.

Among the variety of sensor array types that are used in an electronic system, conducting polymer composites, intrinsically conducting polymers, and metal oxides are often used for the detection of substances in conductivity sensors $[6,16,18,19]$. Despite the drawbacks of polymer composites such as sensor drift, a limit to sensor life, and sensitivity 


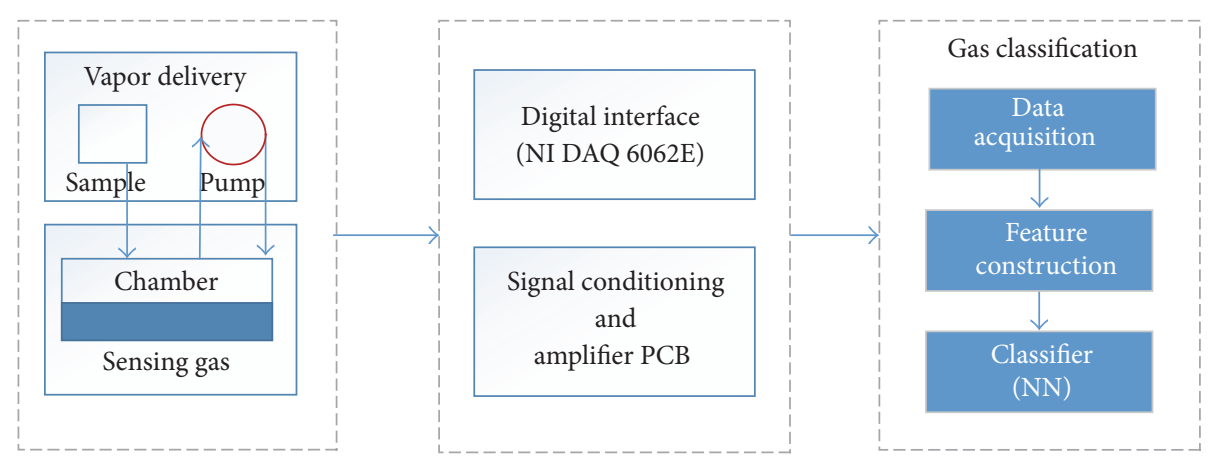

FIgURE 1: Schematic diagram of the e-nose system.

to temperature or humidity, their use has been more frequently compared to other substances [20] because of the corresponding advantages such as a wide range of available polymetric materials, an inexpensive cost, a stable operation capability at room temperature, and a lesser amount of electricity consumption. Sensor measurement largely consists of the following three stages: stabilization, exposure, and purge. Each sensor channel shows different responses depending on the type of gas, and the reactions of each stage are recorded as a single vector $[2,11]$.

Computing systems use a variety of pattern-recognition techniques to discern the data classes that are entered through a sensor array. Gas classification processes can be divided into the following two stages: the extraction of useful features from the gas for classification and the use of the extracted features to design a classifier. Since the data samples that are obtained through the sensor arrays in an electronic system are high-dimensional data that consist of the measured values per sampling point for each channel, a variety of different dimension-reduction methods can be employed to increase the degree of classification accuracy and to effectively and simultaneously reduce the amount of data processing $[2,11,21,22]$. PCA [23], one of the typical dimension-reduction methods, performs classification by projecting data samples on the low-dimensional feature space that allows for the maximal dispersion of the data samples. LDA $[24,25]$ is a method that focuses on finding an optimal linear discriminant function and generally shows a more effective performance compared to PCA when it is applied to classification problems. LDA composes a feature space that narrows distribution within the same class and widens a distance between the means of different classes by using the class information of training data. In $[21,22]$, the two OCA variants CC-PCA and CC-CPCA were used, respectively, in an e-nose system. In $[6,11]$, the CLDA method, which is used to develop the LDA method so that high-dimensional data with high correlations are implemented between the input variables, was used to classify the gas.

The above methods represent the entirety of the measured values from the gas-sample stabilization stage to the purge stage as a single vector and compose a feature space through statistical analysis. The measured value of the sensor array, however, contains different information according to each of the measurement sections, so it could be effective to use the local information together with the global information. In this paper, the combination of the global features that are extracted from the entire measurement section and the local features that are extracted from each local section was therefore used for the gas classification classifier. For this purpose, the ReliefF method [26] was used to evaluate the individual global and local features in terms of the usefulness of not only each feature that classifies the gas but also the combined features that are used for the classifier of an electronic system, whereby only those features with useful classification information were screened.

The proposed method is greatly advantageous in noisy environments because only the features that maintain the discernment information from the variety of sections through the feature selection processes are screened and used, even if the problem occurs during the data measurement process or if noise is mixed with the measured value. The results of the experiment for a variety of volatile organic compound types [20] confirm that the proposed combined features exhibited a sound classification performance in a comparison with the other features that are obtained from the use of the feature extraction method.

This paper is composed as follows: Section 2 explains the discriminant feature extraction method that can be used in an e-nose system and proposes a method for the configuration of combined features that is based on a discriminant analysis, whereby the discernment of the basic features that are extracted from the entire measurement section as well as the local samples sections is measured; Section 3 presents the results of the gas classification experiment; and, finally, Section 4 presents the conclusion.

\section{Construction of Combined Features for Gas Classification}

2.1. Gas Measurement. Figure 1 is a schematic diagram of the e-nose system that is used in this paper; additionally, the micromachined sensor array chips that were used in [20] were used for the gas measurement for this paper. The sensor array of this paper consists of 16 channels that are composed of carbon-black (CB) polymer composite sensors with an interdigitated electrode, microheater, and machined 
TABLE 1: The CB polymer composites used in the sensor array.

\begin{tabular}{ll}
\hline Channel & Polymer \\
\hline 1 & Poly(methyl methacrylate) \\
2 & Polyvinylpyrrolidone \\
3 & Poly(vinyl acetate) \\
4 & Poly(ethylene oxide) \\
5 & Polycaprolactone \\
6 & Poly(4-methylstyrene) \\
7 & Poly(styrene-co-methyl methacrylate) \\
8 & Poly(ethylene-co-vinylacetate) \\
9 & Poly(bisphenol A carbonate) \\
10 & Poly(4-vinyl pyridine) \\
11 & Poly(vinyl butyral)-co-vinyl alcohol-co-vinyl acetate \\
12 & Poly(vinyl stearate) \\
13 & Ethyl cellulose \\
14 & Polystyrene-block-polyisoprene-block-polystyrene \\
15 & Hydroxypropyl cellulose \\
16 & Cellulose acetate \\
\hline
\end{tabular}

membrane in each channel; in Table 1, the 16 kinds of CB polymer composites are indicated. The resistance change of each polymer composite film was observed in response to the chemical-gas combination, and it was recorded every $0.1 \mathrm{sec}$ and measured for a total of $200 \mathrm{sec}$. The entire measurement section can be divided into the following three sections: stabilization ( $30 \mathrm{sec})$, exposure $(60 \mathrm{sec})$, and purge $(1,100 \mathrm{sec})$. First, if a resistance signal is stabilized after a sensor array is placed in the chamber, gas is introduced for $60 \mathrm{sec}$ before it discharges the residual gas, to the outside for the remaining $110 \mathrm{sec}$ through the systemic flow control [27]. The measured data is stored in a PC with the use of the DAQ6062E data acquisition (DAQ) board and LabVIEW (National Instrumentation, USA). A voltage divider operates from $-10 \mathrm{~V}$ to $10 \mathrm{~V}$ and the gain of the 16 identical amplifiers was set to 10 , which is approximately the maximum DAQ resolution [20].

2.2. Discriminant Feature Extraction. Those features that are suitable for classification were extracted by using the LDA method, which is also one of the dimension-reduction methods for a typical classification, whereby gas data that is obtained through the sensor array was involved. When the $n$-dimensional data samples $\mathbf{x}_{k} \in R^{n \times 1}(k=1, \ldots, N)$ that belong to one of the $C$ classes are given, the LDA constitutes a feature space that reduces the scatter of samples in the same class and simultaneously furthers the distance between the class means. For this, the within-class scatter matrix $S_{W}$ and the between-class scatter matrix $S_{B}$ are first defined as follows [24]:

$$
\begin{gathered}
S_{B}=\frac{1}{N} \sum_{i=1}^{C} N_{i}\left(\mu_{i}-\mu\right)\left(\mu_{i}-\mu\right)^{T}, \\
S_{W}=\sum_{i=1}^{C} \sum_{\mathbf{x}_{k} \in c_{i}}\left(\mathbf{x}_{k}-\mu_{i}\right)\left(\mathbf{x}_{k}-\mu_{i}\right)^{T},
\end{gathered}
$$

where $\mathbf{x}_{k}$ refers to the $k$ th sample that belongs to class $c_{i} ; N$ and $C$ are the numbers of the whole samples and classes, respectively; and $\mu_{i}$ and $\mu$ are the sample means that belong to class $c_{i}$ and the whole samples, respectively. Then, the LDA method calculates the projection matrix $W_{\mathrm{LDA}}=$ $\left[\mathbf{w}_{1}^{L}, \ldots, \mathbf{w}_{n^{\prime}}^{L}\right]$ that is made up of the projection vector $\left(\mathbf{w}_{l}^{L}, l=\right.$ $\left.1, \ldots, n^{\prime}\right)$ from $S_{W}$ and $S_{B}$, satisfying the following defined objective function:

$$
W=\arg \max _{W} \frac{\left|W^{T} S_{B} W\right|}{\left|W^{T} S_{W} W\right|},
$$

where $\mathbf{w}_{l}^{L}$ s satisfy $S_{B} \mathbf{w}_{l}^{L}=\lambda_{l} S_{W} \mathbf{w}_{l}^{L}$ that is obtained by calculating the eigenvectors of $S_{W}^{-1} S_{B}$ [24]. In the case of high-dimensional data such as gas data (in this paper, $\mathbf{x}_{k} \in R^{32,000}$ ), however, $n$ generally becomes larger than the rank of $S_{W}(=N-C)$, and it is with respect to the SSS (Small Sample Size) problem [24] that $S_{W}$ becomes singular. As a method to solve this problem, the PCA + LDA method [25] reduces the dimension of the data into $m \leq N-C$ by performing the PCA method prior to the commencement of the LDA method. If the total scatter matrix $S_{T}$ is defined as $S_{T}=\sum_{i=1}^{C} \sum_{\mathbf{x}_{k} \in c_{i}}\left(\mathbf{x}_{k}-\mu\right)\left(\mathbf{x}_{k}-\mu\right)^{T}$, the projection matrix of the PCA + LDA method is $W_{\mathrm{PCA}+\mathrm{LDA}}=$ $W_{\mathrm{LDA}}^{T} W_{\mathrm{PCA}}^{T}$, where $W_{\mathrm{PCA}}=\arg \max _{W}\left|W^{T} S_{T} W\right|$ and $W_{\mathrm{LDA}}$ $=\arg \max _{W}\left(\left|W^{T} W_{\mathrm{PCA}}^{T} S_{B} W_{\mathrm{PCA}} W\right| /\left|W^{T} W_{\mathrm{PCA}}^{T} S_{W} W_{\mathrm{PCA}} W\right|\right)$. If $n^{\prime}(\leq C-1)$ of the projection vectors $\mathbf{w}_{l}^{\mathrm{PL}}$ are selected according to the large eigenvalues among the projection vectors that consist of $W_{\mathrm{PCA}+\mathrm{LDA}}$, the gas data sample $\mathbf{x}_{k}$ is represented as the $n^{\prime}$-dimensional feature vector $\mathbf{y}_{k}$ that consists of the discriminant features $y_{k}$ 's as follows:

$$
\mathbf{y}_{k}=W_{\mathrm{PCA}+\mathrm{LDA}}^{T} \mathbf{x}_{k}=\left[y_{k 1}, y_{k 2}, \ldots, y_{k n^{\prime}}\right]^{T} .
$$

2.3. Combined Feature Configuration Based on a Discriminant Analysis. In the proposed method, the local samples such as $\mathbf{x}_{k}^{\text {st }} \in R^{4,800 \times 1}, \mathbf{x}_{k}^{\text {ex }} \in R^{9,600 \times 1}$, and $\mathbf{x}_{k}^{\text {pu }} \in R^{17,600 \times 1}$ are first obtained by, respectively, separating the measured values that only correspond to the only stabilization, exposure, and purge sections from the global sample $\mathbf{x}_{k}^{\text {tot }} \in R^{32,000 \times 1}$ that contains all of the measured values. The basic feature sets such as $\mathbf{Y}_{k}^{\text {Pool }}=\left[Y_{1}, \ldots, Y_{4(\mathrm{C}-1)}\right]^{T}\left(=\left[y_{k, 1}^{\text {tot }}, \ldots, y_{k, \mathrm{C}-1}^{\text {tot }}, y_{k, 1}^{\text {st }}, \ldots, y_{k, \mathrm{C}-1}^{\text {st }}\right.\right.$, $\left.\left.y_{k, 1}^{\mathrm{ex}}, \ldots, y_{k, \mathrm{C}-1}^{\mathrm{ex}}, y_{k, 1}^{\mathrm{pu}}, \ldots, y_{k, \mathrm{C}-1}^{\mathrm{pu}}\right]^{\mathrm{T}}\right)$ that have an element of $4(C-1)$ number are then composed by extracting the global features $\mathbf{y}_{k}^{\text {tot }}$ and the local features $\mathbf{y}_{k}^{\text {st }}, \mathbf{y}_{k}^{\text {ex }}$, and $\mathbf{y}_{k}^{\mathrm{pu}}$ using (3). To measure the usefulness of each basic feature in terms of the classification of patterns, the ReliefF [26] method, which is effective in selecting useful features to recognize a highdimensional face image like an electronic nose data [28], is used. The ReliefF method can distinguish both useful and nonuseful features depending on how effectively each feature can discern the samples that are near each other; for this purpose, the weighted vector $\mathbf{A}=\left[A_{1}, A_{2}, \ldots, A_{4(\mathrm{C}-1)}\right]^{T}$ that is the value of each feature is defined. For a randomly selected $k$ th sample $\left(\mathbf{x}_{k}\right)$, let the $s$-nearest samples in the same class be $\mathbf{Y}_{k}^{\mathrm{Pool}}$ and let the $s$-nearest samples in the different classes from $\mathbf{Y}_{k}^{\text {Pool }}$ in the feature space be "Hit" $\left(\mathbf{H}_{r}, r=1, \ldots, s\right)$ 


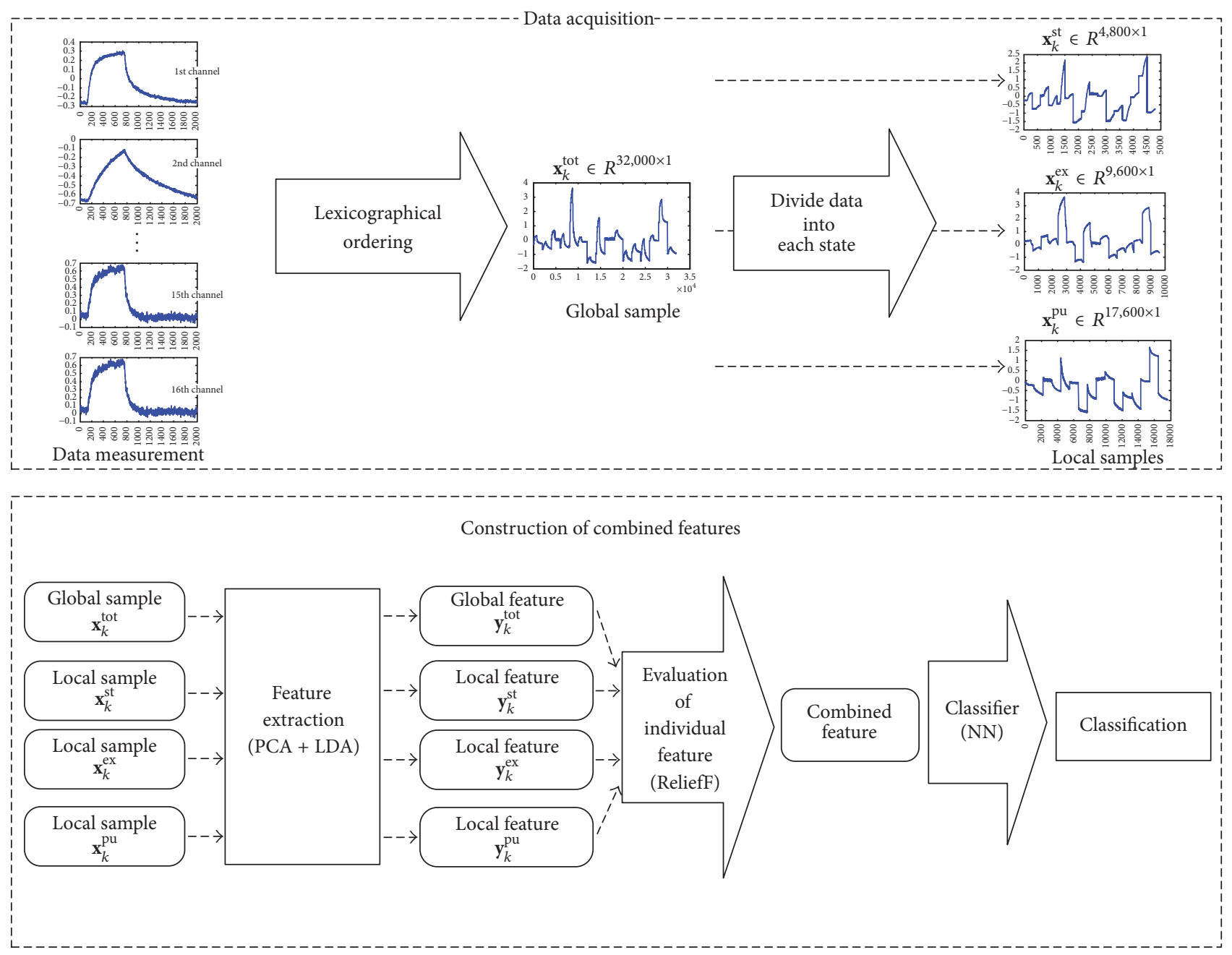

FIGURE 2: Construction process flow of the combined features.

and "Miss" $\left(\mathbf{M}_{r}, r=1, \ldots, s\right)$ [26], respectively. If the $j$ th feature $Y_{j}$ has a different value in both $\mathbf{Y}_{k}^{\text {Pool }}$ and $\mathbf{H}_{r}$, feature $Y_{j}$ separates $\mathbf{Y}_{k}^{\text {Pool }}$ and $\mathbf{H}_{r}$, even though they belong to the same class. This case makes it difficult to discern the class, so the $j$ th element value of the weighted vector $A_{j}$ is reduced. Alternatively, if $Y_{j}$ is in $\mathbf{Y}_{k}^{\mathrm{Pool}}$ and $\mathbf{M}_{r}$, feature $Y_{j}$ thoroughly separates $\mathbf{Y}_{k}^{\text {Pool }}$ and $\mathbf{M}_{r}$ that belong to different classes and increases the value of $A_{j}$. When the initial value of $A_{j}$ is set as zero, the weighted value of feature $Y_{j}$ is updated with training data samples via the following process:

(1) Randomly select sample $\mathbf{x}_{k}$.

(2) Find $\mathbf{H}_{r} s, r=1, \ldots, s$.

(3) Find $\mathbf{M}_{r}\left(c_{l}\right) s=1, \ldots, s$, in the class $c_{l},(l=$ $1, \ldots, C-1)$, which are different from $c_{\mathbf{Y}_{k}^{\text {pool }}}$ in the class of $\mathbf{Y}_{k}^{\mathrm{Pool}}$.

(4) Consider $A_{j}=A_{j}-\sum_{r=1}^{s}\left|Y_{k, j}^{\text {Pool }}-H_{r, j}\right| /(N$. $s)+\sum_{q_{q} \neq c_{\mathbf{Y}_{k}^{\text {Pool }}}}\left[\left(\left(1-P\left(c_{l}\right)\right) /\left(1-P\left(c_{\mathbf{Y}_{k}^{\text {Pool }}}\right)\right)\right) \sum_{r=1}^{s} \mid Y_{k, j}^{\text {Pool }}-\right.$ $\left.M_{r, j} \mid\right] /(N \cdot s)$.
In (4) of the above process, $P\left(c_{l}\right)$ refers to the prior probability of class $c_{l}[26,29]$.

After repeating this process for all of the training data samples $\mathbf{x}_{k}(k=1,2, \ldots, N)$, the final combined feature vector $\left(\mathrm{y}^{\mathrm{CF}}\right)$, which will be used for the gas classification, is composed of features $Y_{j}$ 's that correspond to large $A_{j}$. Through the previously mentioned procedure, we can obtain the combined features that robustly classify a gas even if noise is generated during the gas measurement process, because the unnecessary information in the noisy sections is removed and only the useful features selected from among the global features and local features with a high degree of discernment can be used in the classification process. The entire flow of the proposed method is presented in Figure 2.

\section{Results of the Experiment}

3.1. Experimental Condition. To ensure whether the proposed method effectively classifies gas in an e-nose, the classification performance for the VOC-measurement data includes evaluations of the following eight gas types: acetone, benzene, cyclohexane, ethanol, heptane, methanol, propanol, 


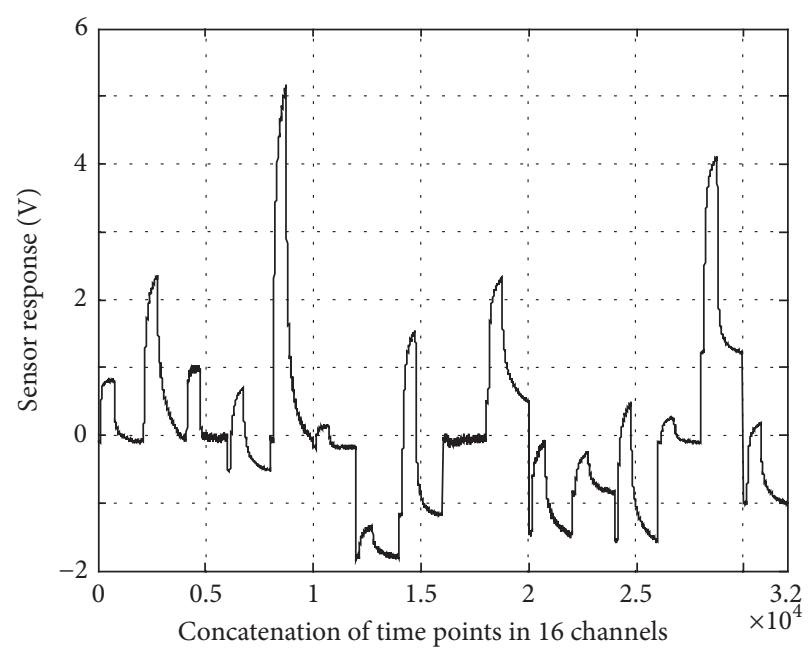

(a)

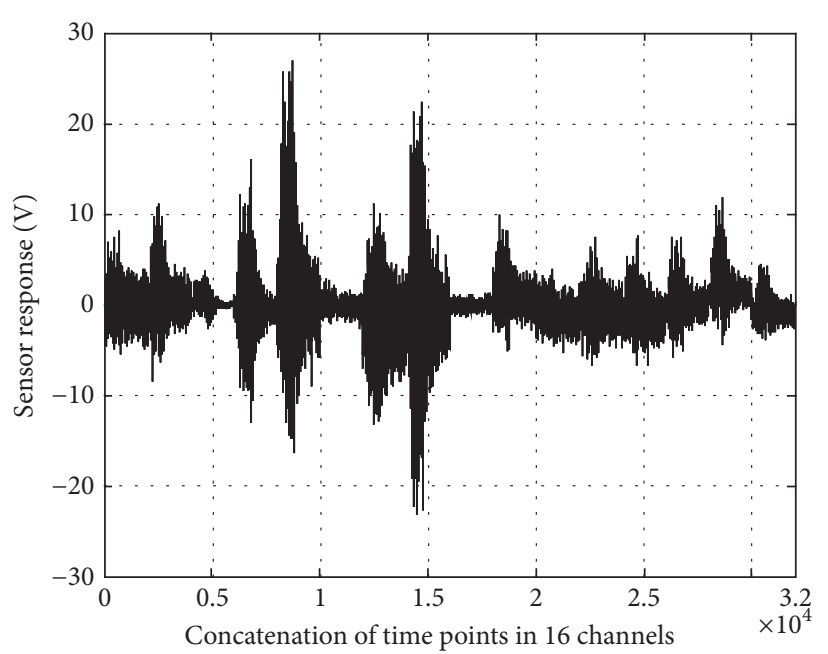

(b)

FIgUre 3: E-nose data without and with Gaussian noise. (a) E-nose data without noise. (b) Data with Gaussian noise (std 3).

and toluene [20]. The total of 160 samples that was used in the experiment comprises 20 collected samples for each type of gas. Each of the samples consists of the values that were measured at the 2,000 time points over $200 \mathrm{sec}$ with a $10 \mathrm{~Hz}$ sampling rate for each channel. The measured values of the 16 channels were saved as the matrix of a $2,000 \times 16$ size before a lexicographic ordering operator was used to convert them into a 32,000-dimensional vector [6]. To determine the effectiveness of the proposed method when noise occurs in the sensing data, the original data samples were mixed with Gaussian noises that have standard deviations of 2, 3 , and 4 , and the performance was evaluated (Figure 3). The classification performance of the 160 data samples was assessed using an eightfold cross-validation strategy [30], whereby a seed-value-based random-number generator was used to mix the 160 samples before they were then divided into eight sections to include samples of the same number. One sample per group, which is a total of 16 samples, was used as the test data and the remaining 140 samples were used as the training data. After performing the experiment eight times in the same way so that all of the data samples of each section were used as test data at least once, the average value of the classification rate was calculated as the final result. In this paper, to increase the statistical reliability of this eightfold cross-validation method, the classification performance was evaluated from the mean value of the results that were obtained by performing the process eight times repeatedly with use of eight different random-seed numbers. All of the data samples were normalized with the mean and standard deviation of the training data to produce a zero mean and a unit standard deviation. When the PCA + LDA method was used to extract the discriminant features, the dimension $(m)$ was reduced by the PCA; the PCA method significantly affects the classification performance of the resultant discriminant features. In this experiment, $m(=105)$ was set to take up $99 \%$ of the entire eigenvalue of $S_{T}$ according to an investigation of the performance evaluation of different $m$ values. The NN (Nearest Neighbor) method was used as a classifier and $l_{2}$ norm was used for the calculation of the distance between two samples [6].

3.2. Classification Rate Evaluation. First, the classification rates of the local features $\left(\mathbf{y}^{\mathrm{st}}, \mathbf{y}^{\mathrm{ex}}, \mathbf{y}^{\mathrm{pu}}\right)$ that were extracted from three types of local samples $\left(\mathbf{x}^{\mathrm{st}}, \mathbf{x}^{\mathrm{ex}}, \mathbf{x}^{\mathrm{pu}}\right)$ were compared. As shown in Figure 4, the noise-free original data shows high classification rates more than $97 \%$ in all of the local features. In Figures 4(b)-4(d), the sensor-based gas concentrates of the local feature of the exposure section show a relatively sound performance compared with the other sections; however, as the degree of noise for all of the local features intensified, the classification rates decreased rapidly. Figure 5 shows the comparison of the classification performances of the proposed combined features $\left(\mathrm{y}^{\mathrm{CF}}\right)$, the FF feature $\left(y^{\mathrm{FF}}\right)$ [2], the PCA + LDA global feature $\left(\mathbf{y}^{\text {tot }}\right)$, the CC-PCA feature $\left(\mathrm{y}_{\mathrm{PCA}}^{\mathrm{CC}}\right)$ [21], and the CPCA feature $\left(y_{C P C A}^{C C}\right)$ [22]. The PCA method was applied to $y_{P C A}^{C C}$ and $y_{C P C A}^{C C}$ once more after the CC-PCA and CC-CPCA methods were performed. As shown in Figure 5(a), each method shows a sound performance more than $96 \%$ in noise-free data; however, as the degree of noise became more severe and while the classification performances of the other methods rapidly decreased, the proposed method maintained more than $90 \%$ of the classification rate, even with a Gaussian noise standard deviation of 4 (Figures 5(b)-5(d)). This maintenance level is due to the effect of the noise that is directly reflected in the classifier input when only global features or local features are used, whereas, in the proposed method, a wide variety of discriminant features were obtained by using global features in combination with a variety of local features. Through the feature selection process, only the features that are less affected by noise and have a sound discernment were selectively used as a classifier input, although some of the features are relatively polluted by noise. By using the 

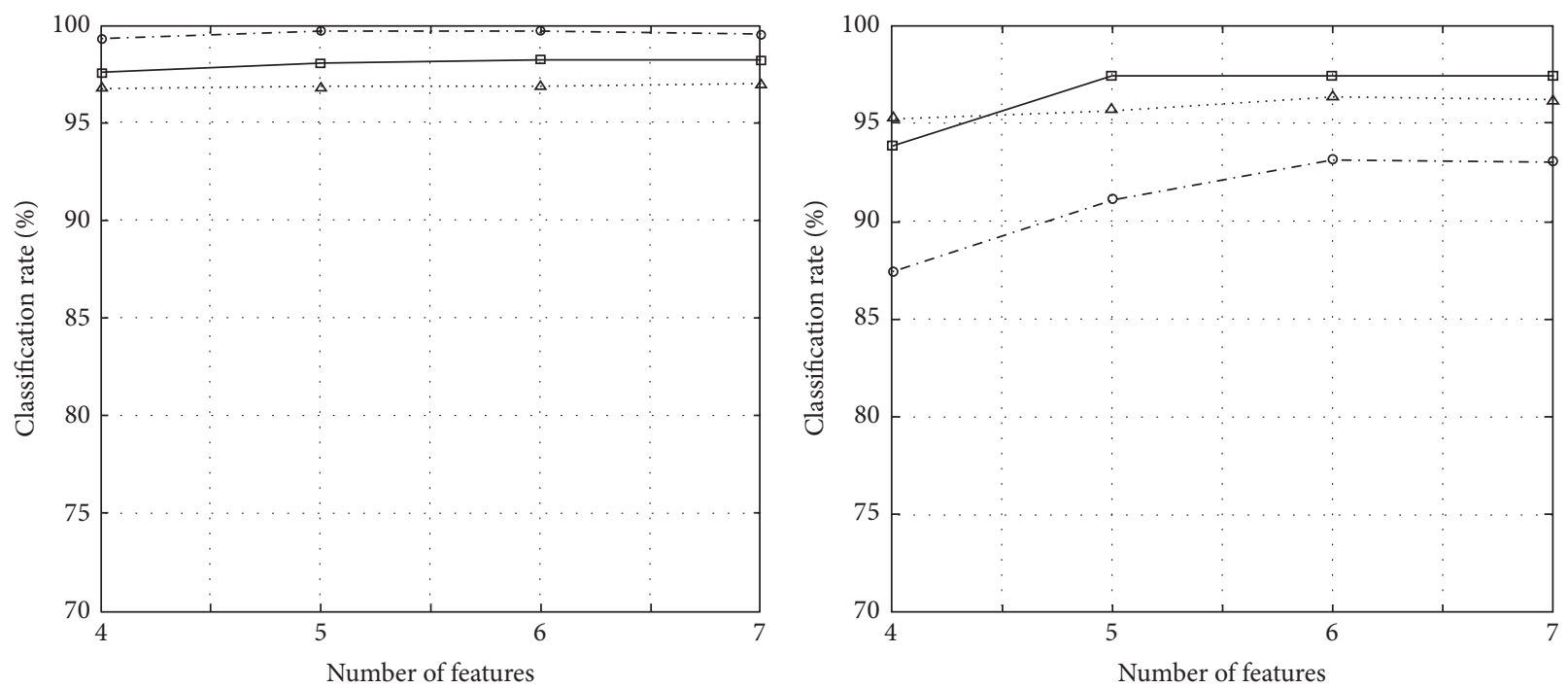

$$
\begin{aligned}
& \cdot \Delta \cdot y^{\text {st }} \\
& \longrightarrow-y^{\text {ex }} \\
& -\circ-y^{p u}
\end{aligned}
$$

(a)

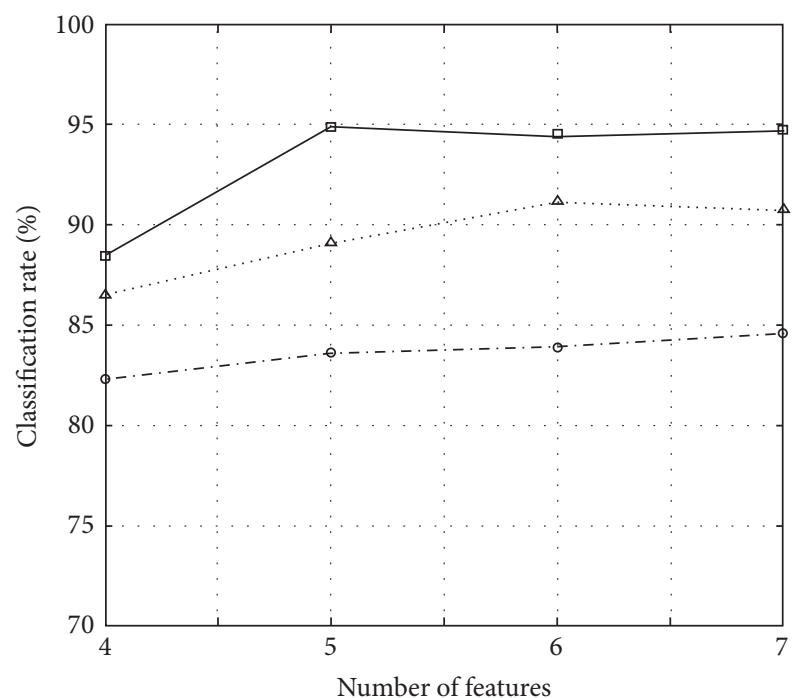

$$
\begin{aligned}
& \cdot \Delta \cdot y^{\text {st }} \\
& \rightarrow-y^{\mathrm{ex}} \\
& \cdot \circ-y^{\mathrm{pu}}
\end{aligned}
$$

$$
\begin{aligned}
& \cdots \Delta \cdot y^{\text {st }} \\
& --y^{\mathrm{ex}} \\
& -0-y^{p u}
\end{aligned}
$$

(b)

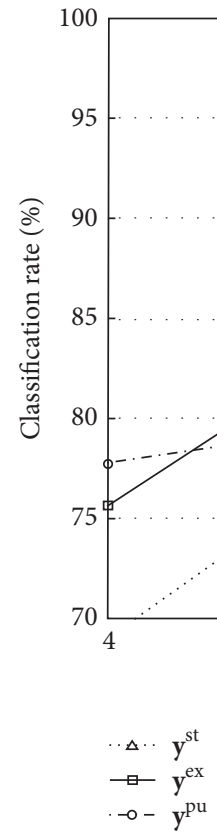

(c)

(d)

Figure 4: Classification rates of local features for the e-nose data. (a) Original e-nose data. (b) E-nose data with Gaussian noise (std 2). (c) E-nose data with Gaussian noise (std 3). (d) E-nose data with Gaussian noise (std 4).

proposed method that operates in the presence of noise more robustly than the other methods, the combined features were consequently configured.

\section{Conclusions}

Gas data measurement that uses sensors in an e-nose system can be classified into several stages according to the process. The sensor response at each stage has different information, from which a variety of features for classification can be extracted. In this paper, three types of local features were extracted in the section that was divided into stabilization, exposure, and purge; these features were then used for the classification, whereby the combined features were configured with the global features that were extracted from the entire section. Based on a discriminant analysis involving the individual global features and the local features, only those features with a large amount of discernment information 


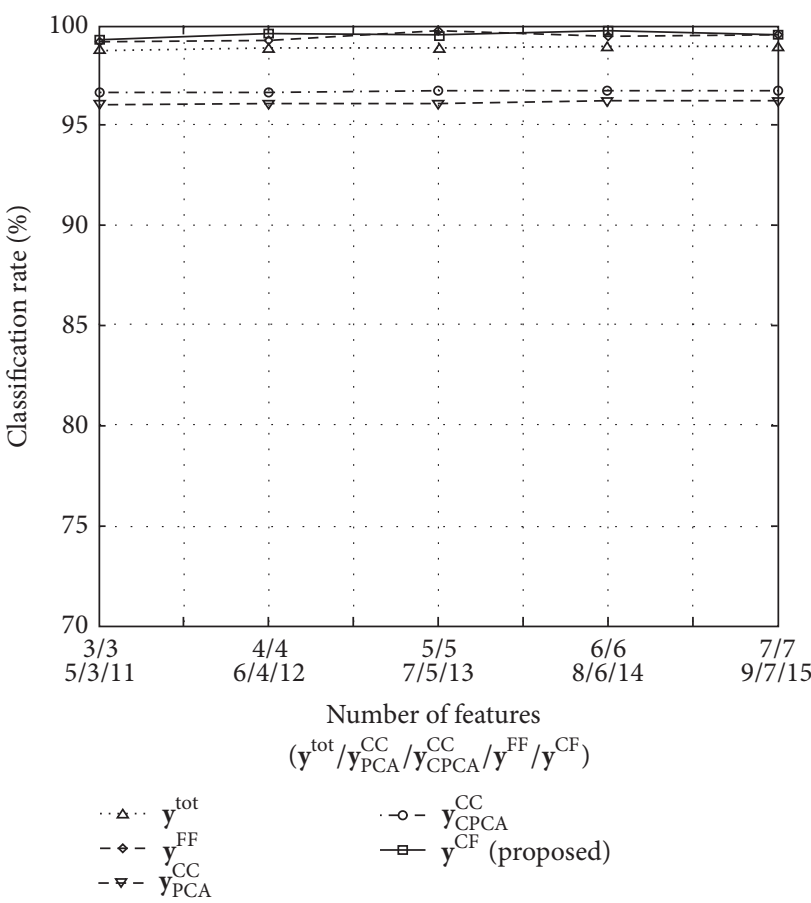

(a)

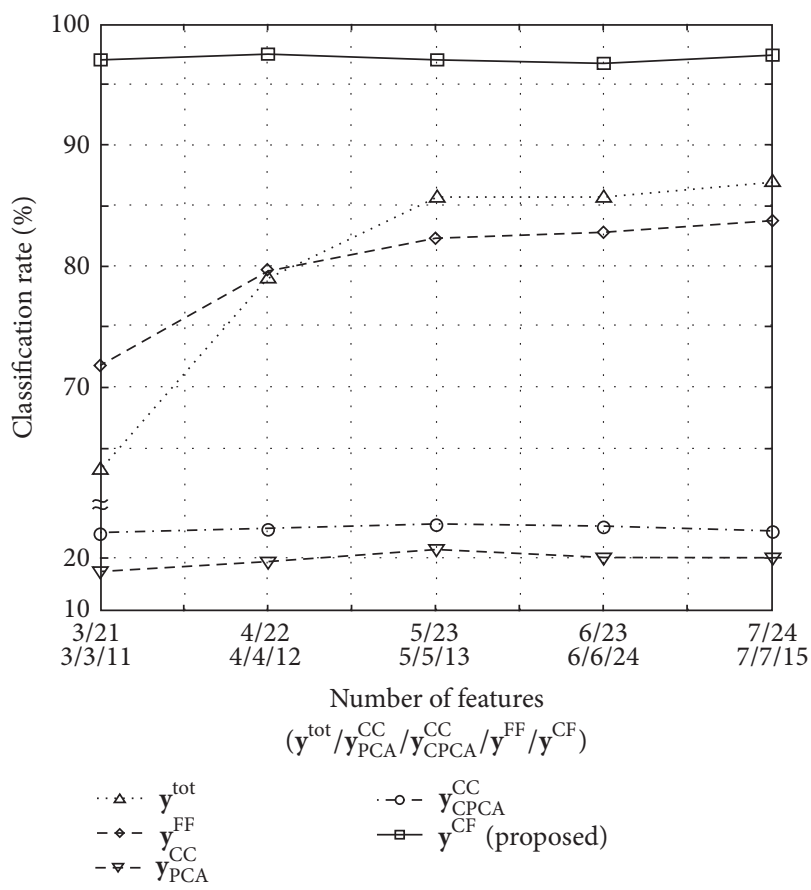

(c)

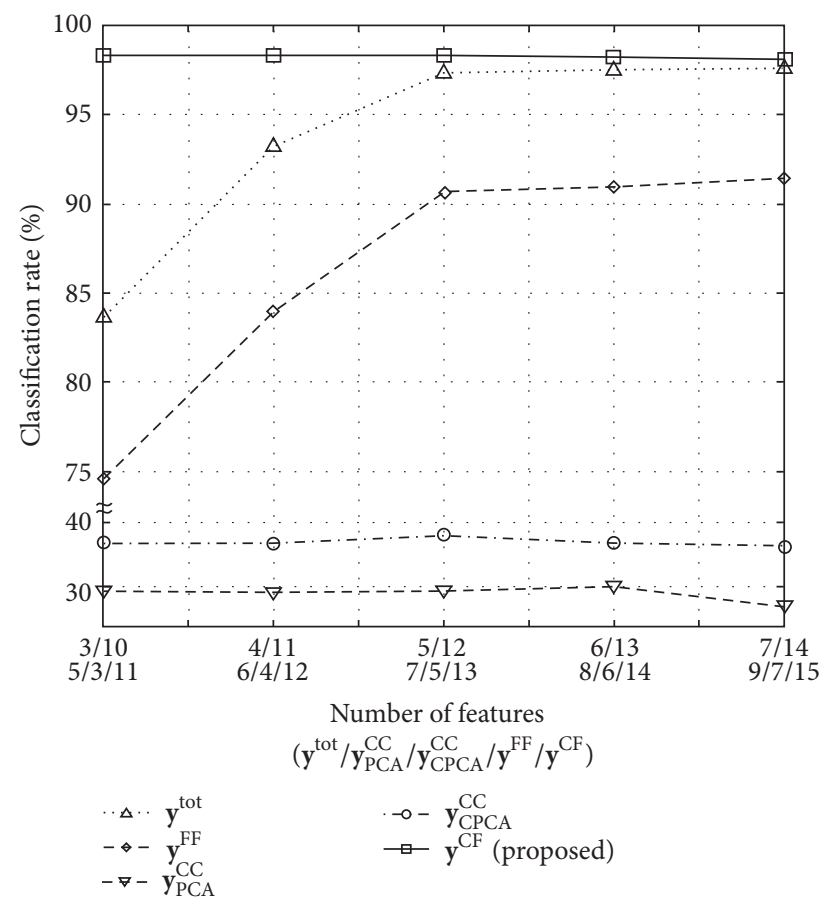

(b)

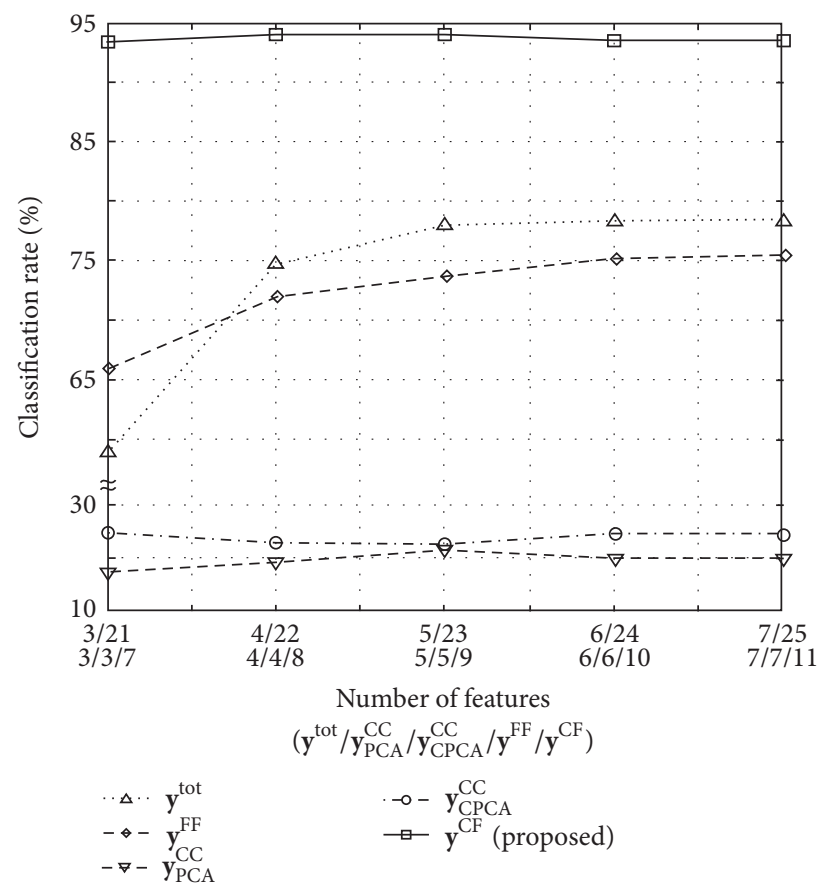

(d)

FIGURE 5: Comparison of classification rates between the proposed method and other methods. (a) Original e-nose data. (b) E-nose data with Gaussian noise (std 2). (c) E-nose data with Gaussian noise (std 3). (d) E-nose data with Gaussian noise (std 4).

were selected, whereby the discernment of each feature was evaluated for the purpose of selection; the selected features were then used to construct the combined features. The proposed method is not limited by a particular feature extraction method. It can be used with a variety of features and the advantages of each feature can be utilized effectively.
For the eight types of gas data that were measured by a sensor array consist of 16 channels, the proposed method showed a sound classification performance; in particular, when noise is generated during the sensing process, the proposed method showed a classification performance that is more effective than those of the other methods. Given the capability to 
test for a variety of complex data, experiments that combine different types of features will be conducted in the future.

\section{Competing Interests}

The authors declare that they have no competing interests.

\section{Acknowledgments}

This work was supported by the Human Resources Program in Energy Technology of the Korea Institute of Energy Technology Evaluation and Planning (KETEP) granted financial resource from the Ministry of Trade, Industry \& Energy, Republic of Korea (no. 20154030200830), and also supported by the Basic Science Research Program through the National Research Foundation (NRF) of Korea funded by the Ministry of Science ICT and Future Planning (no. 2015R1A1A1A05001065).

\section{References}

[1] S.-W. Chiu and K.-T. Tang, "Towards a chemiresistive sensorintegrated electronic nose: a review," Sensors, vol. 13, no. 10, pp. 14214-14247, 2013.

[2] S.-I. Choi, S.-H. Kim, Y. Yang, and G.-M. Jeong, "Data refinement and channel selection for a portable e-nose system by the use of feature feedback," Sensors, vol. 10, no. 11, pp. 10387-10400, 2010.

[3] J. Zhou, T. Feng, and R. Ye, "Differentiation of eight commercial mushrooms by electronic nose and gas chromatography-mass spectrometry," Journal of Sensors, vol. 2015, Article ID 374013, 14 pages, 2015.

[4] M. Bougrini, K. Tahri, Z. Haddi, T. Saidi, N. El Bari, and B. Bouchikhi, "Detection of adulteration in argan oil by using an electronic nose and a voltammetric electronic tongue," Journal of Sensors, vol. 2014, Article ID 245831, 10 pages, 2014.

[5] A. D. Wilson and M. Baietto, "Applications and advances in electronic-nose technologies," Sensors, vol. 9, no. 7, pp. 50995148, 2009.

[6] S.-I. Choi and G.-M. Jeong, "A discriminant distance based composite vector selection method for odor classification," Sensors, vol. 14, no. 4, pp. 6938-6951, 2014.

[7] J. Lerchner, D. Caspary, and G. Wolf, "Calorimetric detection of volatile organic compounds," Sensors and Actuators, B: Chemical, vol. 70, no. 1-3, pp. 57-66, 2000.

[8] M. Farré, L. Kantiani, M. Petrovic, S. Pérez, and D. Barceló, "Achievements and future trends in the analysis of emerging organic contaminants in environmental samples by mass spectrometry and bioanalytical techniques," Journal of Chromatography A, vol. 1259, pp. 86-99, 2012.

[9] Y.-H. Kim and K.-H. Kim, "Ultimate detectability of volatile organic compounds: how much further can we reduce their ambient air sample volumes for analysis?" Analytical Chemistry, vol. 84, no. 19, pp. 8284-8293, 2012.

[10] J. Nicolas, A.-C. Romain, V. Wiertz, J. Maternova, and P. André, "Using the classification model of an electronic nose to assign unknown malodours to environmental sources and to monitor them continuously," Sensors and Actuators, B: Chemical, vol. 69, no. 3, pp. 366-371, 2000.
[11] S.-I. Choi, G.-M. Jeong, and C. Kim, "Classification of odorants in the vapor phase using composite features for a portable Enose system," Sensors, vol. 12, no. 12, pp. 16182-16193, 2012.

[12] C. Di Natale, A. Macagnano, E. Martinelli et al., "Lung cancer identification by the analysis of breath by means of an array of non-selective gas sensors," Biosensors and Bioelectronics, vol. 18, no. 10, pp. 1209-1218, 2003.

[13] W. Khalaf, C. Pace, and M. Gaudioso, "Least square regression method for estimating gas concentration in an electronic nose system," Sensors, vol. 9, no. 3, pp. 1678-1691, 2009.

[14] M. Macías Macías, J. E. Agudo, A. García Manso, C. J. García Orellana, H. M. González Velasco, and R. Gallardo Caballero, "A compact and low cost electronic nose for aroma detection," Sensors, vol. 13, no. 5, pp. 5528-5541, 2013.

[15] A. Norman, F. Stam, A. Morrissey, M. Hirschfelder, and D. Enderlein, "Packaging effects of a novel explosion proof gas sensor," Sensors and Actuators B: Chemical, vol. 95, no. 1, pp. 287-290, 2003.

[16] K. Arshak, E. Moore, G. M. Lyons, J. Harris, and S. Clifford, "A review of gas sensors employed in electronic nose applications," Sensor Review, vol. 24, no. 2, pp. 181-198, 2004.

[17] G.-M. Jeong, N. T. Nghia, and S.-I. Choi, "Pseudo optimization of e-nose data using region selection with feature feedback based on regularized linear discriminant analysis," Sensors, vol. 15, no. 1, pp. 656-670, 2014.

[18] K. J. Albert, N. S. Lewis, C. L. Schauer et al., "Cross-reactive chemical sensor arrays," Chemical Reviews, vol. 100, no. 7, pp. 2595-2626, 2000.

[19] A. Šetkus, A. Olekas, D. Senuliene, M. Falasconi, M. Pardo, and G. Sberveglieri, "Analysis of the dynamic features of metal oxide sensors in response to SPME fiber gas release," Sensors and Actuators, B: Chemical, vol. 146, no. 2, pp. 539-544, 2010.

[20] Y. S. Yang, S.-C. Ha, and Y. S. Kim, "A matched-profile method for simple and robust vapor recognition in electronic nose (Enose) system," Sensors and Actuators B: Chemical, vol. 106, no. 1, pp. 263-270, 2005.

[21] T. Artursson, T. Eklöv, I. Lundström, P. Mårtensson, M. Sjöström, and M. Holmberg, "Drift correction for gas sensors using multivariate methods," Journal of Chemometrics, vol. 14, no. 5-6, pp. 711-723, 2000.

[22] A. Ziyatdinov, S. Marco, A. Chaudry, K. Persaud, P. Caminal, and A. Perera, "Drift compensation of gas sensor array data by common principal component analysis," Sensors and Actuators B: Chemical, vol. 146, no. 2, pp. 460-465, 2010.

[23] M. Turk and A. Pentland, "Eigenfaces for recognition," Journal of Cognitive Neuroscience, vol. 3, no. 1, pp. 71-86, 1991.

[24] K. Fukunaga, Introduction to Statistical Pattern Recognition, Computer Science and Scientific Computing, Academic Press, New York, NY, USA, 2nd edition, 1990.

[25] P. N. Belhumeur, J. P. Hespanha, and D. J. Kriegman, "Eigenfaces vs. fisherfaces: recognition using class specific linear projection," IEEE Transactions on Pattern Analysis and Machine Intelligence, vol. 19, no. 7, pp. 711-720, 1997.

[26] M. Robnik-Šikonja and I. Kononenko, "Theoretical and empirical analysis of ReliefF and RReliefF," Machine Learning, vol. 53, no. 1-2, pp. 23-69, 2003.

[27] S.-C. Ha, Y. S. Kim, Y. Yang et al., "Integrated and microheater embedded gas sensor array based on the polymer composites dispensed in micromachined wells," Sensors and Actuators B: Chemical, vol. 105, no. 2, pp. 549-555, 2005. 
[28] S.-I. Choi, "Construction of composite feature vector based on discriminant analysis for face recognition," Journal of Korea Multimedia Society, vol. 18, no. 7, pp. 834-842, 2015.

[29] S.-I. Choi, J. Oh, C.-H. Choi, and C. Kim, "Input variable selection for feature extraction in classification problems," Signal Processing, vol. 92, no. 3, pp. 636-648, 2012.

[30] L. Liu and M. T. Zsu, Encyclopedia of Database Systems, Springer, New York, NY, USA, 2009. 


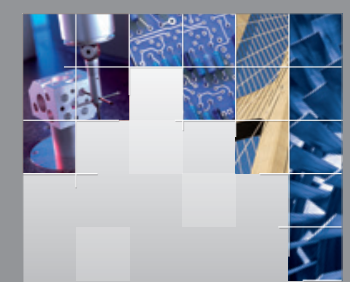

\section{Enfincering}
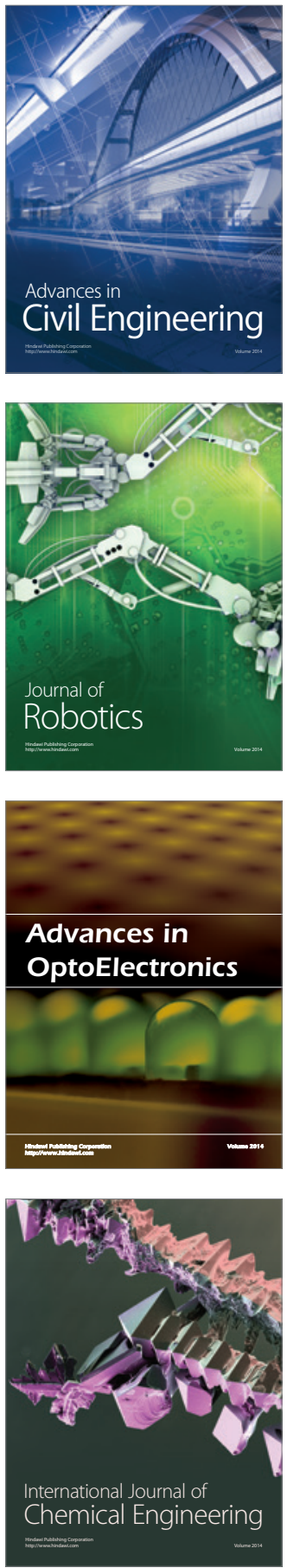

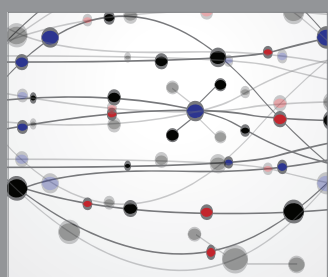

The Scientific World Journal

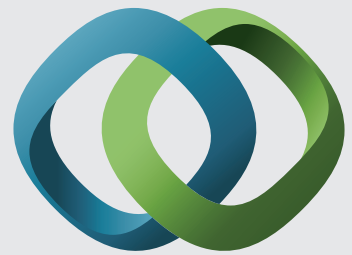

\section{Hindawi}

Submit your manuscripts at

http://www.hindawi.com
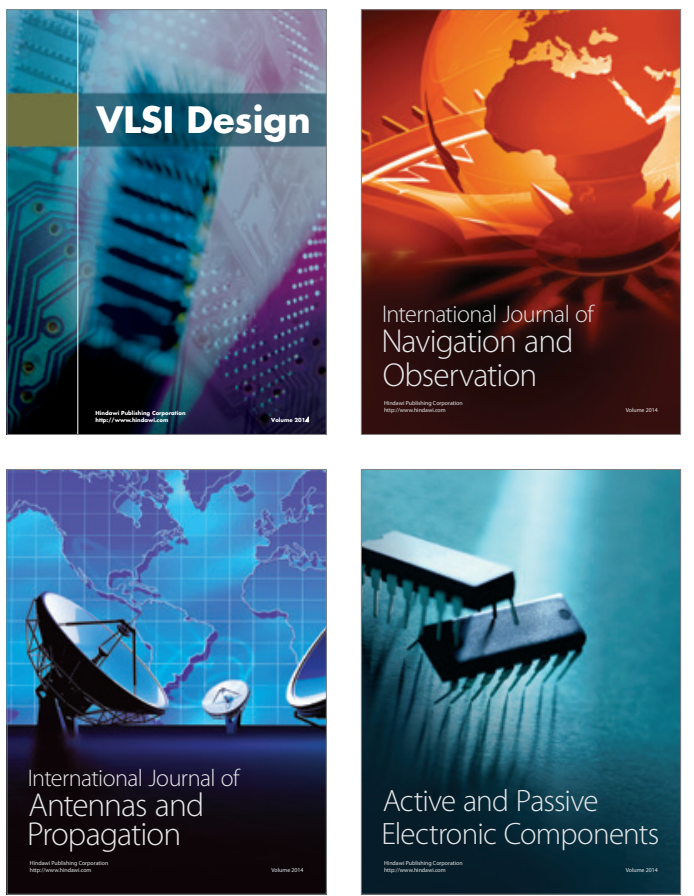
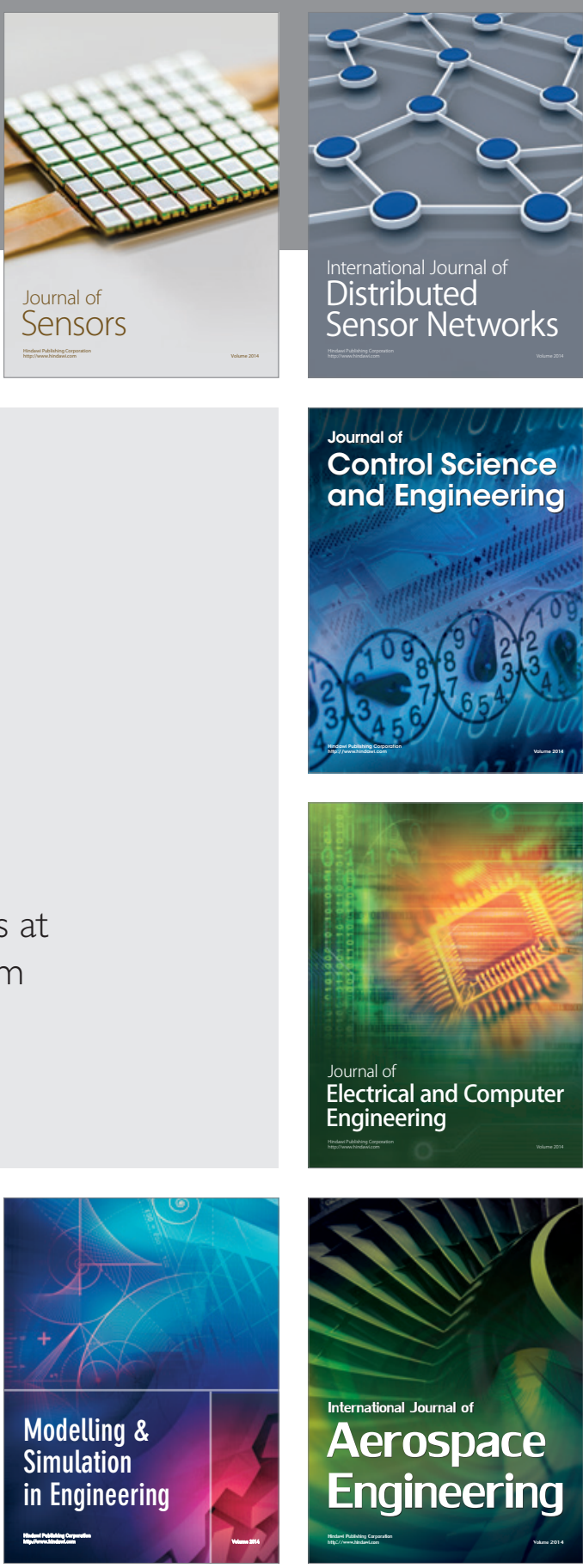

International Journal of

Distributed

Sensor Networks

Journal of

Control Science

and Engineering
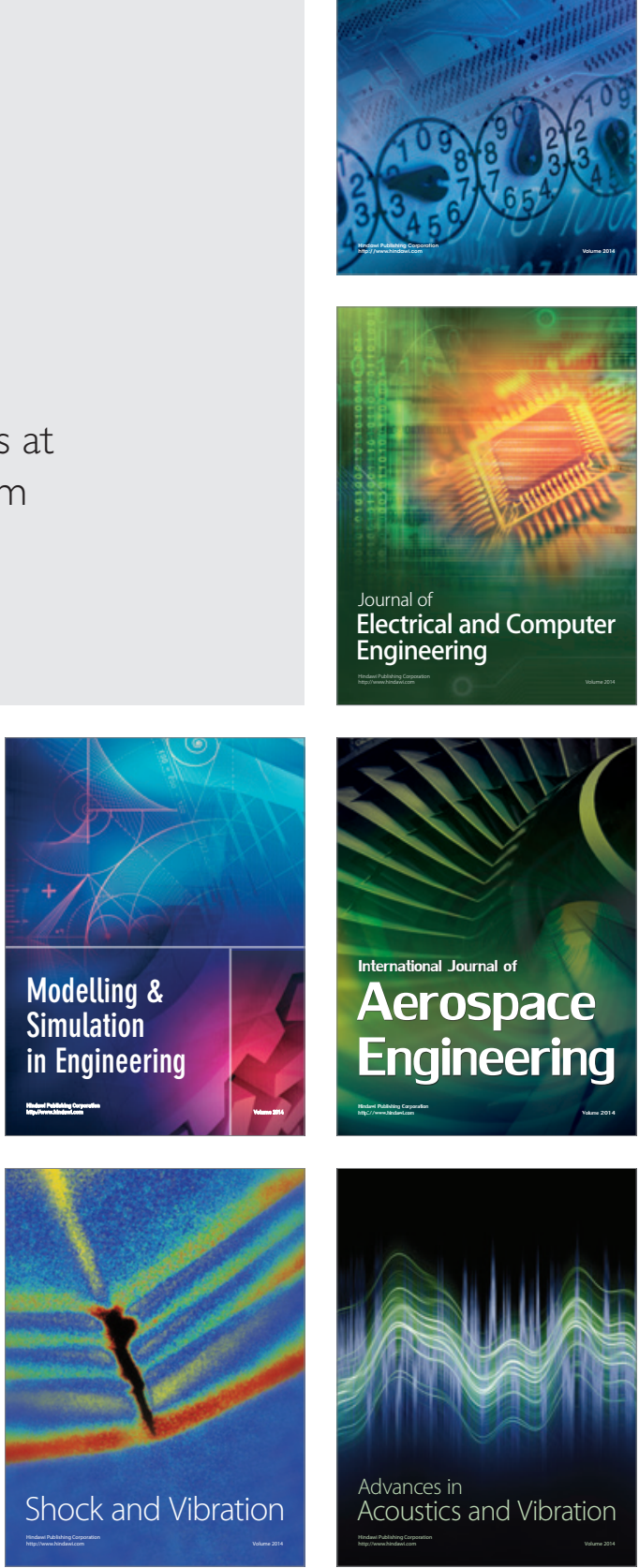